Noname manuscript No.

(will be inserted by the editor)

\title{
Recent Evidence for Convection in Sunspot Penumbrae
}

\author{
Göran B. Scharmer
}

Received: date / Accepted: date

\begin{abstract}
Whereas penumbral models during the last 15 years have been successful in explaining Evershed flows and magnetic field inclination variations in terms of flux tubes, the lack of contact between these models and a convective process needed to explain the penumbral radiative heat flux has been disturbing. We report on recent observational and theoretical evidence that challenge flux tube interpretations and conclude that the origin of penumbral filamentary structure is overturning convection.
\end{abstract}

Keywords First keyword · Second keyword · More

\section{Introduction}

Sunspot magnetic fields and dynamics have been studied scientifically for 100 years. Despite considerable progress during the last decade, a theoretical framework that explains sunspot fine structure, dynamics, magnetic fields and energy balance in a consistent manner is only now beginning to emerge. This situation can partly be attributed to the small horizontal scales associated with sunspot fine structure and the relatively poor spatial resolution achieved with spectropolarimetric observations. In addition, realistic numerical 3D MHD simulations of sunspots have only recently become possible.

During the last few years, there has been a remarkable improvement in the quality and diversity of observational data relevant to the understanding of sunspot fine structure, dynamics, magnetic fields and energy balance. In particular, high-spatial resolution observations from the Swedish 1-m Solar Telescope (SST) and the Solar Optical Telescope (SOT) on Hinode reveal new sunspot structure and flow patterns at odds with prevailing interpretations in terms of flux tube models. In addition, theoretical arguments as well as recent 3D MHD simulations of sunspot fine structure underline problems of these interpretations and lead to the conclusion that the origin of penumbral fine structure is overturning convection.

G.B. Scharmer

Institute for Solar Physics, Royal Swedish Academy of Sciences, AlbaNova University Center, SE-10691 Stockholm

E-mail: scharmer@astro.su.se 
In the present review, we describe recent progress in our understanding of penumbral fine structure and put that in context with existing models. Rather than attempting to summarize the extensive literature on penumbrae, we discuss selected key papers and attempt to describe their interconnections and to critically review conclusions drawn. We also point out connections between observed penumbral fine structure and magnetic flux concentrations outside sunspots, such as faculae. We hope to convince the reader that the new picture emerging is one of improved consistency as regards observations and theory of sunspot penumbrae in particular, but also with respect to umbral dots, light bridges and faculae.

\section{Overview of Established Models}

For the past 15 years, the predominant paradigm of penumbral filaments has been based on nearly horizontal flux tubes, portrayed and modeled as radially aligned cylinders, embedded in a more vertical magnetic field. These flux tube interpretations have their roots in the work of Mever \& Schmidt (1968) who proposed to explain Evershed flows as siphon flows, originating from a difference in magnetic field strength between the two foot points of a flux tube. In the 70's, flux tubes and clusters of flux tubes were also established in models of magnetic flux concentrations, surrounded by field-free gas, with scales ranging from less than $100 \mathrm{~km}$ to that of a large sunspot.

\subsection{Embedded flux tubes}

Of particular importance in the current sunspot literature is the uncombed penumbra model, proposed by Solanki \& Montavon (1993) to explain the strongly asymmetric Stokes $\mathrm{V}$ profiles observed on the limb side penumbra for sunspots away from disk center. This model addressed an apparent problem of very strong line-of-sight (LOS) gradients in the inclination angle of the penumbral magnetic field, inferred from Stokes data by Sanchez Almeida \& Lites (1992). The large LOS inclination gradients derived from this data were (incorrectly, see Sect. 4) interpreted to imply volume currents and associated curvature forces strong enough to completely disrupt static force balance in the spot (Sanchez Almeida \& Lites 1992; Solanki et al. 1993). The uncombed penumbra model avoids this problem, at least partly, by postulating the existence of discrete flux tubes, within which the magnetic field is assumed to be homogeneous and therefore current-free. The uncombed model thus 'replaces' smooth inclination gradients and (assumed) large volume currents with discontinuous changes at the boundary of the flux tube and an associated current sheet. Solanki and Montavon demonstrated that a nearly horizontal flux tube, with a strong flow parallel to its magnetic field, can explain the observed net circular polarization (NCP) resulting from Stokes V asymmetries of this configuration. Moreover, if such a flux tube is located entirely above the photosphere, both its upper and lower boundaries contribute with the same sign to the asymmetry of its Stokes V profile, thus enhancing the NCP.

Various implementations of flux tube models with polarized radiative transfer were later developed by e.g. Martínez Pillet (2000); Schlichenmaier \& Collados (2002); Borrero et al. (2007); Borrero (2007); Tritschler et al. (2007); Bellot Rubio et al. (2004, 2003). These calculations demonstrate consistency between the calculated azimuthal variation of $\mathrm{NCP}$ and measurements made at low spatial resolution in visible and near 
infrared spectral lines. Two-component inversions interpreted within the context of embedded flux tube models and applied to low spatial resolution (0.6-1 arcsec) Stokes data by Borrero et al. (2004, 2005, 2006) similarly were shown to be largely consistent with the assumed inversion (flux tube) model.

\subsection{Siphon flow and dynamic flux tube models}

The first flux tube model proposed to explain Evershed flows in penumbrae is the siphon flow model of Meyer \& Schmidt (1968). In this model, a difference in field strength between the two footpoints of a flux tube leads to a difference in gas pressure, driving a flow in the direction of the footpoint with the highest field strength. This work was later followed up by Degenhardt $(1989,1991)$ and Thomas $(1988)$ and the model further refined in a series of papers by Montesinos \& Thomas (1989, 1993, 1997); Thomas \& Montesinos (1990, 1991). Given the free parameters of the calculations, the siphon flow model of Montesinos \& Thomas (1997) allow consistency with the discovery that the Evershed flow connects to patches of opposite magnetic polarity at deep layers near the outer boundary of a sunspot (Westendorp Plaza et al. 1997).

Siphon flow models allow an interpretation of Evershed flows as steady flows, with the properties of the flow determined by assumed conditions at the footpoints of the flux tube and of the surrounding atmosphere. The mechanism that produces the magnetic field strength difference between the footpoints, needed to generate a gas pressure gradient to drive the flow, is not explained by such models. Jahn \& Schmidt (1994) proposed the concept of interchange convection of magnetic flux tubes (or rather, sheets) as an explanation of the penumbral heat flux. To investigate this proposal, Schlichenmaier et al. (1998a b) developed a simplified 1D, one-component numerical model of such a flux tube and studied its time evolution. In this model, a flux tube initially in contact with the magnetopause (the outer boundary of the sunspot) is heated radiatively by the external field-free gas. Its subsequent evolution is driven by the buoyancy of the flux tube and the superadiabatic stratification of the surrounding penumbra atmosphere, assumed to have properties unaffected by the flux tube. At the surface, radiative cooling of the tube causes it to loose buoyancy such that its upper part settles at a height of about $100 \mathrm{~km}$ above the photosphere. A gas pressure gradient, driving the Evershed flow, develops along the tube from downstream radiative cooling.

Later simulations by Schlichenmaier (2002, 2003) with reduced numerical viscosities show a similar initial behavior of the flux tube. However, near the surface, the flux tube subsequently develops standing waves downstream from the footpoint with downflows diving down into the convectively unstable layers beneath the surface. The crests of this oscillating flux tube remain visible above the surface and show an inward migration in the inner part of the umbra and an outward migration in the outer penumbra and outside the penumbra. This behavior is similar to that of observed penumbral grains in the inner and outer penumbra and moving magnetic features outside the penumbra. The discovery of small-scale bipolar magnetic features propagating from the mid penumbra to outside the penumbra, where they become moving magnetic features, is consistent with the 'sea serpent' behavior of Schlichenmaier's moving flux tubes (Sainz Dalda \& Bellot Rubio 2008).

A problem, investigated by several authors, e.g., Solanki et al. (1993); Rezaei et al. $(2006)$, is the large radial mass flux of the Evershed flow inside the penumbra. Only part of this flow appears to continue in the magnetic canopy above the quiet sun 
photosphere outside the spot. To explain this, most of the Evershed flow must submerge close to the outer boundary of the penumbra. The moving tube model simulations show such downflows within the penumbra. However, Thomas (2005, 2006) objected that the undulations seen in the 'sea serpents' of Schlichenmaier (2002) should occur preferentially in the horizontal plane and hence can explain neither moving grains nor convective downflows.

The question of a heating mechanism to explain the penumbral radiative heat flux was investigated by Schlichenmaier \& Solanki (2003). Based on estimates of the radiative cooling time and the time span of successive emergences of flux tubes, they concluded that interchange convection cannot provide the needed energy flux. Weiss et al. (2004) also argued against interchange convection on the basis that long loops of magnetic field connecting to a distant active region cannot possibly interchange with horizontal fields carrying Evershed flows.

The conclusion of Schlichenmaier \& Solanki (2003) was that upflows along the magnetic flux tubes can explain the penumbral brightness, but only if the flux tube submerges again within a distance of $1000-2000 \mathrm{~km}$ from their footpoint. The upflow in a narrow tube cannot supply the radiative energy losses over a distance corresponding to the entire radial extent of a penumbra unless it submerges and is re-heated. Such re-heating does not solve the energy flux problem, however, since it relies on a (convective) mechanism to transport the heat to the bottom of the flux tube. Nevertheless, the discovery of field lines returning to the penumbra and associated downflows (Westendorp Plaza et al. 1997) was considered as support for this explanation (Schlichenmaier \& Solanki 2003).

\subsection{Convection and downward pumping of magnetic flux}

The siphon flow model of Montesinos and Thomas is unrelated to any convection process operating in the penumbra. This model represents a stationary solution that cannot explain time dependent behavior such as moving penumbral grains (Thomas 2006). These grains are instead interpreted as originating from a moving convective pattern in the brighter parts of the penumbra (Weiss 2002, 2006a b). Whereas the moving tube simulations show localized downflows inside and outside the penumbra, the arched flux tubes of the siphon flow model require a mechanism to submerge and hold down their outer parts to sustain equilibrium (Montesinos \& Thomas 1997). Thomas et al. (2002a) and Weiss et al. (2004) proposed that this submergence of the flux tubes occurs as the result of downward pumping by convection outside the sunspot. They even took this proposal one step further and proposed that this downward pumping is the origin of the filamentary structure of the penumbra. In this view, the salient features of penumbrae: their filamentary structures, the strong variations in magnetic field inclination across filaments and the Evershed flows, are to a large extent explained by what happens outside the sunspot. Magnetic fields in bright and dark filaments are distinct and cannot be interchanged (Thomas \& Weiss 2004). To support this, Thomas \& Weiss (2004); Weiss et al. (2004); Weiss (2006b) refer to X-ray observations and TRACE images showing loops extending over great distances across the Sun. We believe that their description and connection to the interlocking comb structure of the penumbra is misleading. Virtually all information about large fluctuations in the magnetic field inclination within the penumbra comes from spectral lines formed within a few hundred $\mathrm{km}$ above the photosphere. The images referred to (Sams et al. 1992) do not have 
the spatial resolution needed to separate X-ray loops (interpreted to outline field lines) from bright and dark filaments. As far as the author knows, there are no observations that allow us to conclude that azimuthal variations in the magnetic field inclination associated with filamentary structures correspond to field lines that are widely separated also far away from the penumbral photosphere. We have argued (Spruit \& Scharmer 2006) that the strong inferred variations in the magnetic field inclination within the first one or two hundred $\mathrm{km}$ above the penumbral photosphere, seen even in the inner penumbra, cannot be explained by a mechanism operating outside the sunspot. Instead, these strong variations suggest a local mechanism at work. The bright and dark filaments are not distinct and they can interchange. Furthermore, Evershed flows are associated with field lines that only locally and during a limited time are nearly horizontal.

\section{Limitations and problems of flux tube interpretations}

The success of the uncombed penumbra model (Solanki \& Montavon 1993) in explaining observed Stokes spectra and net circular polarization (NCP) (Borrero et al. 2007; Borrero 2007; Tritschler et al. 2007; Bellot Rubio et al. 2004, 2003) is unquestionable. Furthermore, the moving tube simulations of Schlichenmaier (2002) make excellent contact with the uncombed penumbra model. It demonstrates consistency with observed strong upflows in bright grains (Rimmele \& Marino 2006) and the behavior of bright grains and moving magnetic features (Sainz Dalda \& Bellot Rubio 2008). It is hardly surprising that the ability of these models to explain azimuthal and line-of-sight (LOS) gradients of the penumbral magnetic field and Evershed flows was deemed successful.

However, a fundamental problem remains. Whereas the embedded flux tubes simulated by Schlichenmaier are consistent with observations in many respects, such flux tubes present problems in explaining penumbral heating Spruit \& Scharmer 2006; Scharmer \& Spruit 2006). As discussed above, a horizontal flux tube is likely to heat the penumbra over a radial distance not much more than $1000 \mathrm{~km}$ (Solanki \& Montavon 1993; Schlichenmaier 2003), which is typical of a penumbral grain rather than a penumbral filament. Even over such a short distance, radiative cooling of the flow leads to significant temperature and brightness gradients along the flux tube unless there is a separate source of heating below the flow channel (Schlichenmaier et al. 1999). Spruit and Scharmer therefore argued that the presence of flux tubes covering a large fraction of the penumbral surface would constitute a hindrance for heating of the penumbra. They also pointed out that the existence of elevated flux tubes extending up to a few hundred $\mathrm{km}$ above the penumbral photosphere correspond to unlikely perturbations in a magnetic field so dominant already at this height that it must be expected to be nearly potential. Furthermore, the moving tube simulations represent a highly idealized model that cannot not be expected to be more than a coarse representation of reality. In particular:

- The existence of the flux tube is an assumption in the model

- The model is 1-dimensional and the flux tube assumed to be 'thin' (see below)

- The simulations correspond to a 1-component model with the properties of the background atmosphere unaffected by the evolution of the flux tube

- Only a single flux tube is simulated. The influence of neighboring flux tubes is not accounted for. 
- The curvature forces of the surrounding magnetic field are ignored and its influence is reduced to a scalar magnetic pressure, similar to a gas pressure.

The same objections apply to the siphon flow models discussed in previous sections.

In view of these short-comings, it is remarkable that the moving tube simulations appears to capture important properties of penumbral dynamics. This is further discussed in Sect. 4.4.

Magnetostatic flux tube models including forces from a surrounding potential magnetic field (Borrero 2007) demonstrate the difficulties of embedded flux tube configurations. Prescribing a specific (circular) cross section for the flux tube corresponds to an overconstrained problem such that not only the gas pressure but also the temperature and density within the flux tube are given by force balance alone. There is thus no room for an energy equation with this type of models. Furthermore, equilibrium is not possible with a purely potential magnetic field inside the flux tube. In the models shown there is an azimuthal component, corresponding to a volume current aligned with the flux tube, in addition to the radial field component. The bottom part of the flux tube is nearly evacuated whereas the top part is denser than the surroundings in order to balance the magnetic forces at the top and bottom, stretching and flattening the flux tube. These problems originate from the surrounding magnetic field wrapping around the flux tube and cannot be resolved by making the flux tube thinner.

In attempt to understand the temperature structure and energy balance of penumbral flux tubes, Ruiz Cobo \& Bellot Rubio (2008) developed a model for a flux tube with a weak magnetic field aligned with a homogeneous magnetic field along the flux tube axis. An objection against this model is that it suffers from a lack of consistency as regards force balance, which is implemented in a way that is equivalent to ignoring the vector properties of the surrounding magnetic field. The origin of this problem is the same as that of the models of Borrero (2007): Prescribing the shape of the flux tube cross section is in general incompatible with either force balance or an energy equation.

\subsection{Ambiguities of interpretations based on inversions}

A major obstacle to understanding penumbra fine structure has been the lack of adequate spatial resolution in observed polarized and unpolarized spectra. In spite of successful adaptive optics systems operating on major solar telescopes, it has not been possible to reach the diffraction limit with the long integration times needed for such data with adequate signal-to-noise. (However, by combining many short exposure frames and using image restoration techniques, filter-based systems allow near diffraction limited spectropolarimetry van Noort \& Rouppe van der Voort 2008; Scharmer et al. 2008a).) The exception is observations in the near infrared, for example those made with the Tenerife Infrared Polarimeter, TIP (Martínez Pillet et al. 1999) on the German Vacuum Tower Telescope (VTT) at wavelengths around 1.5 $\mu$. At that wavelength, the diffraction limited resolution is about 0.6 arc sec with the VTT.

At a spatial resolution of 0.6 arc sec or worse, penumbral fine structure is not adequately resolved. To compensate for this and in order to test the validity of the embedded flux tube (uncombed penumbra) model, various observers, e.g., (Borrero et al. 2004, 2006; Bellot Rubio et al. 2003, 2004) have implemented two-component inversion techniques to interpret Stokes spectra. These investigations show that it is indeed possible to fit the data with the assumed (highly idealized) representations of 


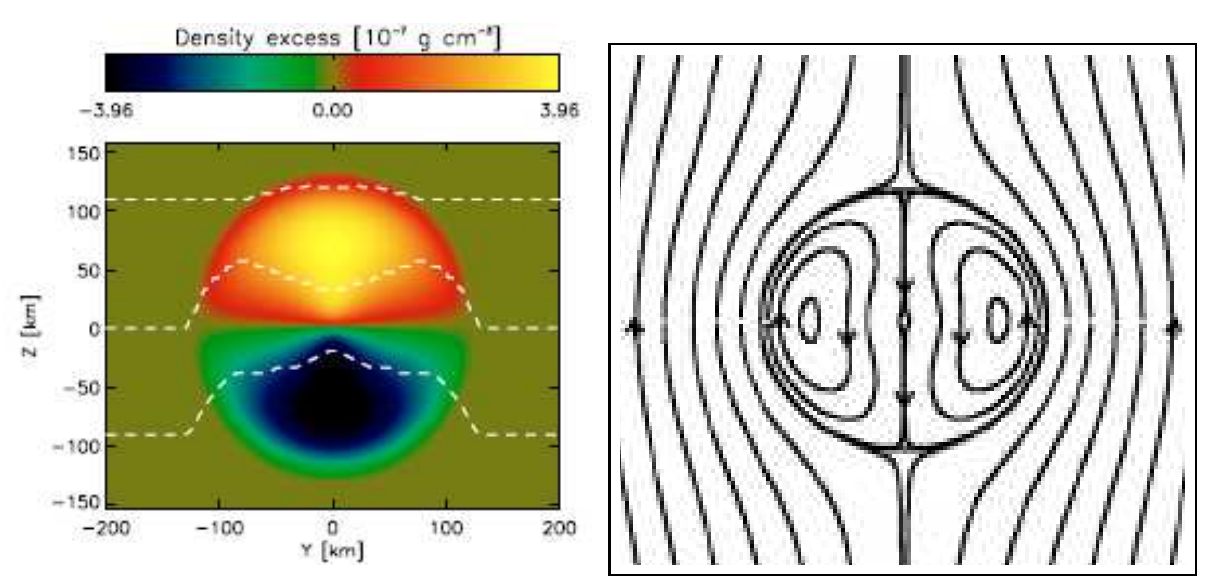

Fig. 1 Left: Magnetostatic flux tube model of Borrero (2007) with dashed curves showing (top to bottom) optical depths $\tau$ of $0.1,1$ and 10 . Note that only the upper part of the flux tube is located above the photosphere and that nearly the entire flux tube is located below $\tau=0.1$. Right: Transverse field lines of a similar (but not identical) model (Borrero et al. 2007). Note the similarity of the magnetic field configuration above the flux tube in this model and that of the convective gap models, shown in Fig 4

flux tubes. Forward calculations of Stokes spectra (Martínez Pillet 2000) and unpolarized spectra (Rimmele 1995; Bellot Rubio et al. 2006) based on flux tube models also demonstrate consistency. In some cases, it was demonstrated also that the observed data could equally well be reproduced with flux tube representations and models with smooth gradients (Martínez Pillet 2000; Rouppe van der Voort 2002; Borrero et al. 2004; Bellot Rubio et al. 2006). This ambiguity is a consequence of the width of the radiative transfer response function, smearing out the effects of discontinuities in the observed (Stokes) spectra. The interpretations of Stokes spectra clearly show compatibility with flux tube interpretations. However, the simplicity of the implemented inversion models and the use of two components to represent observational data of penumbral fine structure at inadequate spatial resolution adds to these uncertainties to the extent that we are justified in questioning whether a description in terms of embedded flux tubes is an adequate representation of penumbra fine structure.

\section{Convective origin of penumbral filaments}

An alternative explanation to understanding penumbra fine structure was proposed by Spruit \& Scharmer (2006). The filamentary structure is explained by convection in radially aligned (nearly) field-free gaps just below the visible surface. Such intrusions unavoidably lead to strong variations in the magnetic field strength and inclination above the gaps, but these variations are fully consistent with even a simple potential magnetic field configuration. The model explains dark cores in bright filaments Scharmer et al. 2002), seen in the inner and mid penumbra (c.f., Fig.s 2 and 3) as an indicator of strong field strength variations across filaments, leading to a strongly varying Wilson depression. The Evershed flow is in this model identical to the horizontal flow component of the convection (Scharmer et al. 2008b). We explain this model in more details in the following. 


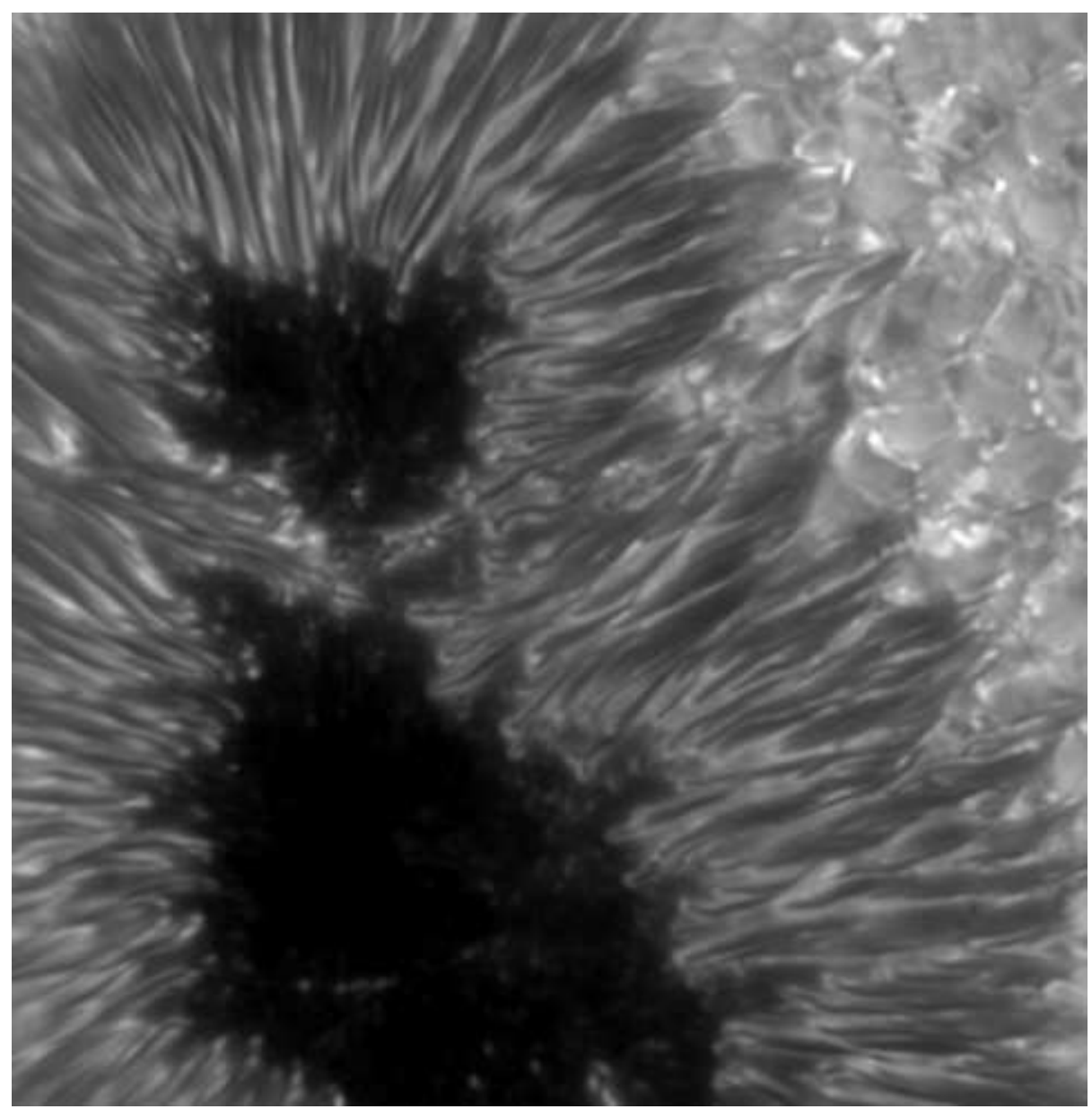

Fig. 2 Sunspot located at a heliocentric distance of $20 \mathrm{deg}$, observed with the SST on 12 Sep 2006 (van Noort \& Rouppe van der Voort 2008). The image shows the Stokes I intensity, averaged over the blue and red wings of the 6302 iron line. Note the dark cores, clearly visible in the inner penumbra.

The large inclination gradients are a consequence of magnetic fields being divergence free $(\nabla \cdot \mathbf{B}=\mathbf{0})$ : field lines cannot disappear at the top of a gap (or flux tube) but must bend around it. To estimate the characteristic vertical scale $H$ for these inclination variations, we can assume a potential magnetic field (Spruit \& Scharmer 2006). For filaments separated by a distance $L$, this gives $H \approx L / 2 \pi$. With a typical separation of 1 " between filaments, this corresponds to a vertical height scale of $120 \mathrm{~km}$. Simple magnetostatic models for such configurations, based on identical temperature variations with height for the two components, show distinct differences between the inner penumbra, where the magnetic field is more vertical and stronger than in the outer penumbra (Scharmer \& Spruit 2006). In the inner penumbra, the magnetic field is cusp-shaped above the gaps and associated with a large $(\approx 200-300 \mathrm{~km})$ Wilson depression relative to that of the gaps. Even when the temperature is the same inside 


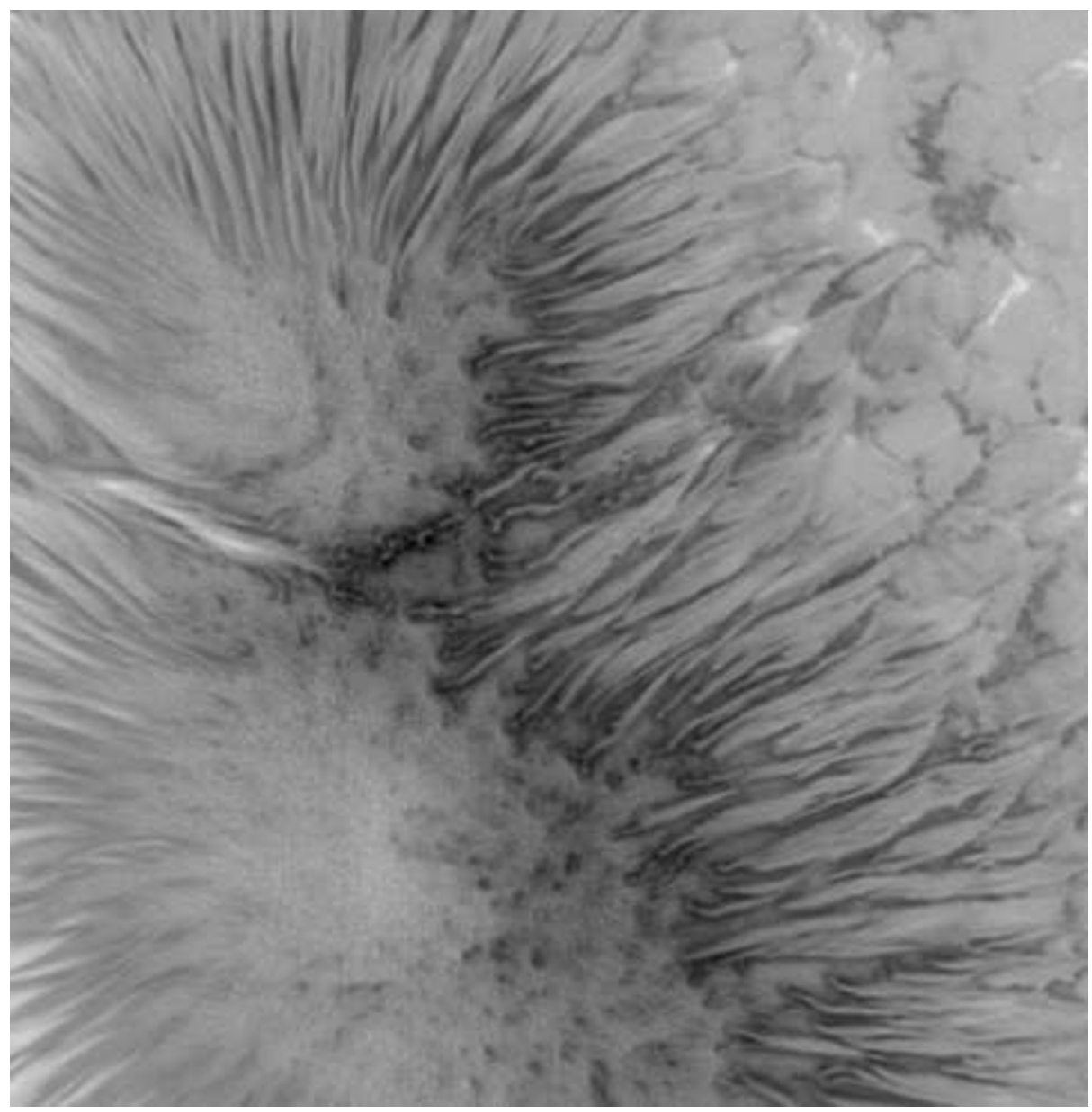

Fig. 3 The same sunspot as shown in Fig 2 (van Noort \& Rouppe van der Voort 2008). The image shows the difference between Stokes V (circularly polarized light), recorded in the blue and red wings of the 6302 iron line. This serves as a proxy for the line-of-sight component of the magnetic field vector. Note the strongly reduced polarization signal at locations of the dark cores, suggesting strongly reduced field strength at these locations.

and outside the gaps, the strong Wilson depression leads to an observed brightness that is lower above the gaps than between the gaps. The dark-cored filaments discovered with the Swedish 1-m Solar Telescope (SST) (Scharmer et al. 2002), seen in the inner and mid penumbra, are thus explained by a combination of increased opacity associated with a strongly reduced field strength and an overall drop of temperature with height (Spruit \& Scharmer 2006). In the outer penumbra, the Wilson depression is only on the order of $50 \mathrm{~km}$ in these models. With such a small Wilson depression, intensity variations from 'global' vertical temperature gradients cannot be expected to completely dominate over local horizontal and vertical gradients associated with details of the heating and cooling of convecting gas. In the outer penumbra we therefore do not expect the same kind of relation between filament brightness and field strength as for 

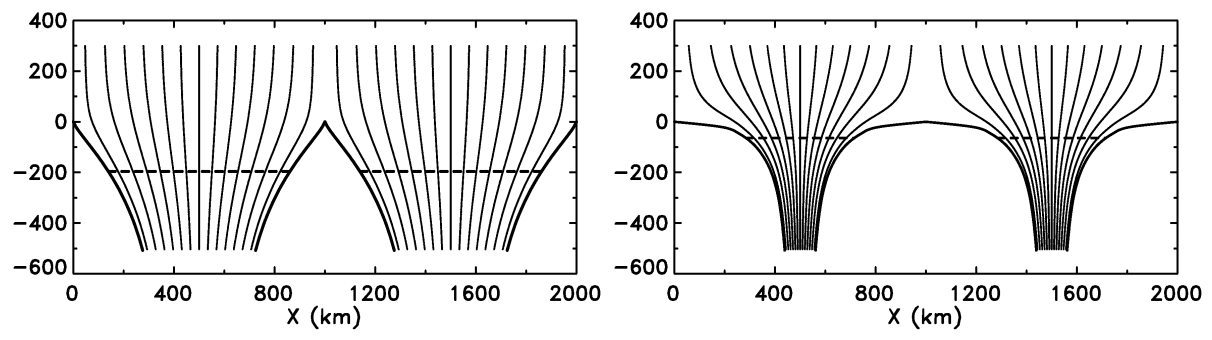

Fig. 4 Magnetostatic convective gap models (Scharmer \& Spruit 2006) for the inner (left) and outer (right) penumbra. The dashed curves show the height at which the gas pressure in the magnetic component equals the photospheric gas pressure in the field-free component. This serves as proxy for the $\tau=1$ surface and leads to Wilson depressions on the order of $200 \mathrm{~km}$ for the inner and $50 \mathrm{~km}$ for the outer penumbra. Note the similarity of the magnetic field configuration for the outer penumbra above the 'photosphere' in this model and the flux tube model, shown in Fig 1

the inner penumbra. The magnetostatic models are therefore in qualitative agreement with the absence of dark cores in the outer penumbra.

The potential magnetic field configurations associated with two magnetostatic models are shown in Fig 4 The upper figure shows the calculated field lines for a weak (1000 G) nearly horizontal (average inclination $75 \mathrm{deg}$ ) magnetic field and the lower figure for a stronger $(1800 \mathrm{G}$ ) and more vertical (average inclination $45 \mathrm{deg}$ ). Also shown are the shapes of the gap and the height at which the gas pressure between the gaps is equal to that of the field-free component at $z=0$ (horizontal dashed lines). This serves as a proxy for the height at which the continuum optical depth is equal to unity. As is clear from the figure, the magnetic field configurations above the gaps are associated with strong gradients. The simple potential field model thus explains large magnetic field inclination variations above the penumbral photosphere without invoking forces in these layers. The associated current sheet is located at and below the photosphere, where the gas pressure is much higher than a few hundred $\mathrm{km}$ above the photosphere. This is in contrast to the uncombed model (Solanki \& Montavon 1993), where the strong gradient in the magnetic field is a direct consequence of a local perturbation in the form av an embedded flux tube located above the photosphere. The current sheets associated with such flux tubes are difficult to combine with magnetostatic equilibrium because of the lower gas pressure at these heights (Spruit \& Scharmer 2006). Indeed, most of flux tube in the magnetostatic model of Borrero (2007) is buried below the photosphere and only about $130 \mathrm{~km}$ protrudes above the surrounding photosphere. It seems very difficult, if not impossible, to construct similar models for flux tubes located entirely above the penumbral photosphere.

The convective gap model thus explains strong magnetic field gradients above the penumbral photosphere as a necessary consequence of potential fields. This model eliminates the problems of large curvature forces discussed by Sanchez Almeida \& Lites (1992); Solanki et al. (1994), constituting a corner-stone argument in favor of the uncombed penumbra model (Solanki \& Montavon 1993). The convective gap model also predicts configurations for the inner and outer penumbra that are quite different. In the inner penumbra, the field is cusp-shaped and associated with a large Wilson depression, large field strength fluctuations but relatively small fluctuations in inclination. In the outer penumbra, the magnetic field is spine-like (Lites et al. 1993), with a small Wil- 


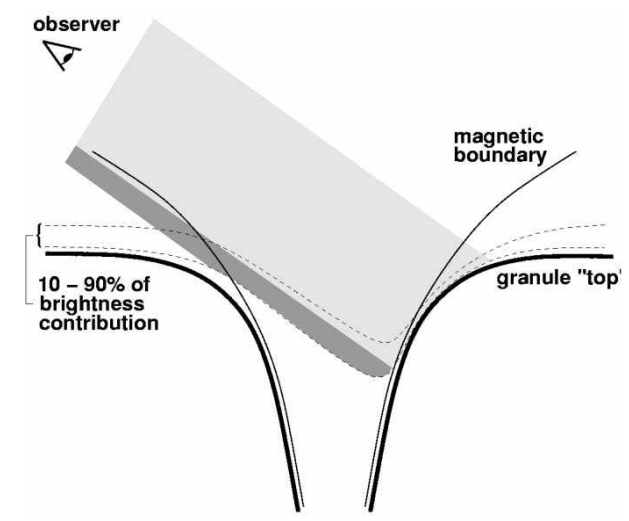

Fig. 5 Schematic drawing explaining the brightness of faculae observed near the limb (Keller et al. 2004). The low gas pressure associated with the strong magnetic field of the flux concentration makes it essentially transparent such that we can see the hot granular wall of the surrounding denser field-free gas. The optical depth unity surface is strongly tilted with respect to the horizontal by the shape of the flux tube magnetic field such that we see deeper in to the hot convecting granules close to the limb than at disk center, explaining the brightness. A similar situation occurs with penumbral filaments associated with strong variations in field strength (see Fig 4). Another similarity between flux tubes and faculae is that the radiative cooling of the surrounding field-free gas through the facula leads to a convective flow pattern that is downward adjacent to the facula and that this downflow is observable on the limb-side but hidden from view on the center-side of the facula. In a similar way, strong Wilson depressions associated with narrow penumbral filaments lead to obscuration of the limb-sides of the filaments already for small heliocentric distance. Convection in such filaments are also affected by radiative cooling through the surrounding magnetic gas such that we expect upflows in the middle of the filaments and downflows at their sides.

son depression, and small field strength fluctuations above the photosphere but with large inclination variations. These qualitative differences between the inner and outer penumbra are in good agreement with observations (Scharmer \& Spruit 2006).

The overturning convective flow patterns associated with the gaps are predicted to be upward in the middle and downflow along the boundaries to the magnetic components (Scharmer \& Spruit 2006). Added to this flow pattern is a radially outward (Evershed) flow, explained by Scharmer et al. (2008b) on the basis of 3D MHD simulations (Heinemann et al. 2007) as being identical to the horizontal component of this convection. In our model, the dark cores of the penumbral filaments correspond to locations of convective upflows, in contradiction with what we expect from field-free convection. As explained above, the strong fluctuations in field strength across the filamentary structures in the inner penumbra lead to a correlation between brightness and field strength, such that we see deeper in to the hotter gas where the field strength is high. The mechanism for producing the dark penumbral cores is directly related to the mechanism that produces bright faculae, proposed initially by Spruit (1976) and well established by numerical simulations (Keller et al. 2004; Carlsson et al. 2004; Steiner 2005) and high-resolution SST observations (Lites et al. 2004). In the case of faculae, convection occurs in the surrounding darker and field-free photosphere, whereas convection is inhibited or strongly suppressed in the brighter and magnetic faculae.

The connection between dark-cored filaments and faculae can be carried further, c.f., Fig. 5 The brightness of faculae near the limb is explained by the $\tau=1$ sur- 
face being strongly inclined to the horizontal at the limbside of faculae (Spruit 1976; Keller et al. 2004; Carlsson et al. 2004). Looking through the nearly transparent gas within the strong magnetic field, we see deeper into the surrounding hot convecting gas close to the limb than at sun center. In a similar way, we see deeper into the convecting parts of the penumbral filaments when viewing sunspots well away from disk center and at +/- 90 degrees from the symmetry line (Scharmer \& Spruit 2006). The convective gap model leads us to interpret the absence of observational evidence for penumbral convection as not only due to lack of spatial resolution: there is also a difficulty of seeing deep enough into the filaments to be able to observe the convection for sunspots close to disk center. In addition, there is the confusion from the brightness - field strength correlation already discussed above.

\subsection{Limitations of the convective gap model}

The magnetostatic gap models discussed above (Scharmer \& Spruit 2006) predict a gradual transition from cusp-shaped magnetic fields in the inner penumbra to spinelike (Lites et al. 1993) magnetic field configurations in the outer penumbra, in good agreement with observations. However, these simple models assume a perfectly fieldfree gap and do not include an energy equation, nor are the forces associated with the convective flows included. Details of flows and their interactions with the magnetic field cannot be explained with this simple model. The explanation of dark cores relies on opacity effects that are obvious only for the model corresponding to the inner penumbra. We expect these results to be relatively robust. In the outer penumbra, predictions about filamentary brightness are more difficult without an energy equation. Furthermore, observations show strong Evershed flows in the outer penumbra where the magnetic field is weaker. This combines to making the kinetic energy density $\rho v^{2} / 2$ of comparable magnitude to the magnetic energy density $B^{2} / 2 \mu_{0}$ such that we expect relatively strong effects from the flow on the magnetic field. Whereas the magnetostatic gap models show good overall agreement with observed properties of penumbral magnetic fields, we cannot expect detailed agreement between the gap model and observations also in the outer penumbra. Only more accurate models and numerical simulations can provide this.

\subsection{Support from observations}

The interpretation of light bridges as essentially field-free gaps dividing the umbra of a sunspot in two parts (Leka 1997; Jurčák et al. 2006) does not seem controversial. 3D MHD simulations of such structures (Nordlund 2006; Heinemann 2006) reproduce observed dark lanes running along the center of such structures (Lites et al. 2004) and demonstrate that the origin of this dark structure is the same as proposed for the convective gap model. Of considerable importance is therefore that dark-cored light bridge structures occasionally show smooth transitions to dark-cored penumbral filaments (Langhans 2006; Scharmer et al. 2007), strongly suggesting similar origin. Upflows in light-bridge dark lanes and the dark cores of penumbral filaments (Rimmele 2008 ) suggest a common interpretation in terms of convection, but with evidence for horizontal flows at greater heights also fitting a flux tube interpretation. Connections 

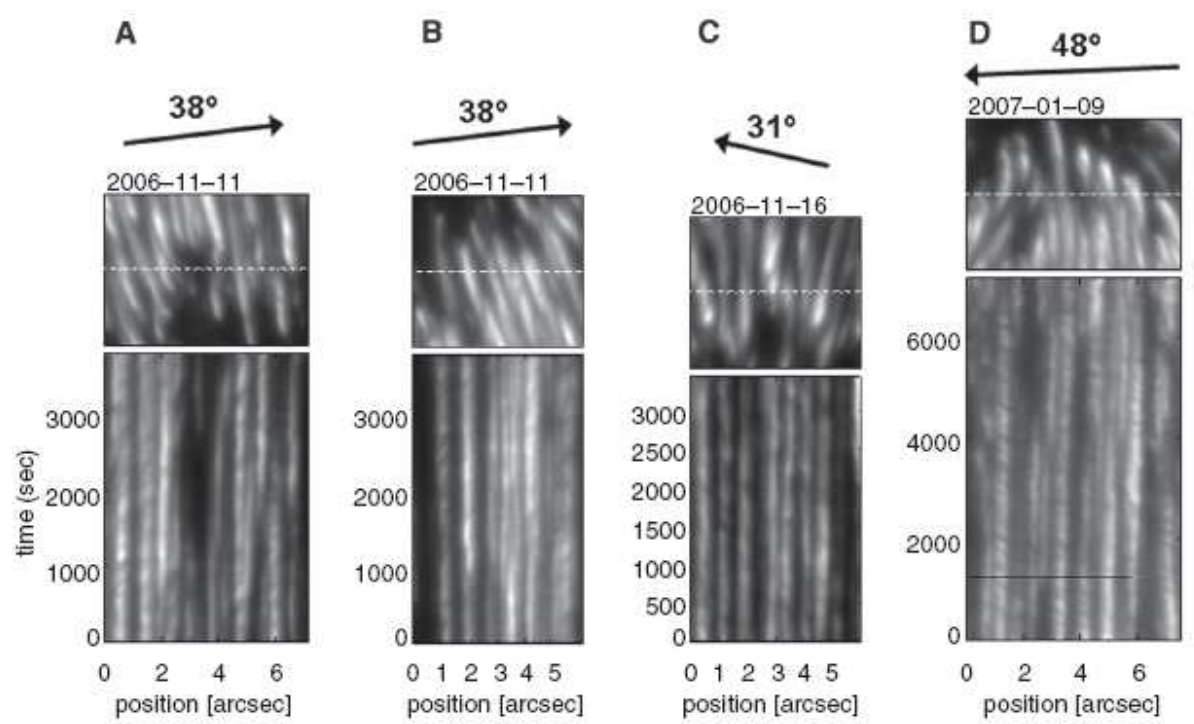

Fig. 6 Examples of space-time slices across filaments in the inner penumbra observed with SOT/Hinode (Ichimoto et al. 2007). The disk center direction is indicated with an arrow and labeled with the corresponding heliocentric distance. The upper image of each pair shows the surroundings of the cut across the filaments (dashed line) analysed in the space-time slices (lower image).

of dark-cored penumbral filaments to peripheral umbral dots and dark cores in light bridges have been reported also by Bharti et al. (2007b).

Several recent papers report evidence for convection in umbral dots (Bharti et al. 2007a; Rimmele 2008; Riethmüller et al. 2008). Although this provides no direct evidence for penumbral convection, the direct connection of peripheral umbral dots to dark-cored filaments (Langhans 2006; Langhans et al. 2007; Rimmele 2008) provides 'circumstantial' evidence for this interpretation. We caution, however, that the umbral dots observed are much larger than those simulated by Schüssler \& Vögler (2006) and in some cases resemble granular intrusions.

In a paper of fundamental importance, Ichimoto et al. (2007) find strong evidence for overturning penumbral convection by analyzing continuum images and spectra observed with SOT on Hinode. Space-time plots created along lines crossing filaments in the inner penumbra, at directions $+/-90 \mathrm{deg}$ from the line connecting the center of the solar disk and the center of the sunspot, displayed twisted ropelike structures. The 'twisting' motion was consistently in the direction toward sun center for both sides of the spot and irrespective of whether the spot was East or West of the meridian. (c.f, Fig(6). The apparent twist observed is therefore neither an actual twist nor a helical motion of individual filaments but must be a viewing angle effect. The interpretation Ichimoto et al. 2007) is of upflows of overturning convection, viewed from the side. With the limb-side part of these filaments hidden from view, such flows will always appear to be in the direction of sun center direction for spots observed away from disk center. This is in perfect agreement with the predictions of the magnetostatic gap model for the inner penumbra (Scharmer \& Spruit 2006). Here the Wilson depression 
between the gaps is predicted to be so large that the limbside part of the filaments is invisible already for disk center distances in the range 21-35 deg. The spot observed by Ichimoto et al. was located at a disk center distance of 31-48 deg. The flow pattern observed is consistent with that predicted by Scharmer \& Spruit (2006).

Based on high-resolution SST data, Zakharov et al. (2008) recently inferred similar evidence of convective flows for a sunspot located $40 \mathrm{deg}$ from disk center. Surprisingly, this is interpreted in terms of convective rolls (Danielson 1961). However, the horizontal flow toward the center of the filament at the bottom of such a roll, needed to verify its existence, is unobservable. Furthermore, the life times of penumbral filaments are on the order of 1 hour or more (Langhans et al. 2007). To sustain the radiative output over such a long time, the convective upflow must persist to depths much larger than a few hundred km. Finally, the observations of Ichimoto et al. (2007) were made in the inner penumbra, where the magnetic field has a strong vertical component, whereas roll-like convection is expected to be primarily associated with more horizontal magnetic field.

\subsection{Support from 3D MHD simulations}

In contrast to what is to what is the case for faculae (Keller et al. 2004; Carlsson et al. 2004) and umbral dots (Schüssler \& Vögler 2006), realistic simulations of entire sunspots have not yet been feasible. This is partly due to the difficulties of thermally relaxing such a deep structure and maintaining its stability but mostly due to the huge range of scales associated with a fully developed sunspot. The first attempts to carry out 3D MHD simulations with radiative energy transfer of fine structure in a small sunspot were carried out by Heinemann et al. (2007) and further discussed by Scharmer et al. (2008b). Recently, simulations using a similar setup and grid separation, but with a much larger computational box, were carried out by Rempel et al. (2008). A synthetic continuum image calculated from these simulations is shown in Fig. 7 The approach taken in both simulations is to reduce the computational effort by using a rectangular computational box containing only a small 'azimuthal' slice of a sunspot. The overall results of these simulations obtained with two independent codes are quite similar, although differing strongly in the length of the penumbral filaments:

- The origin of filamentary structures is overturning convection and the dark cores are caused by a locally elevated $\tau=1$ surface, supporting the convective gap model (Spruit \& Scharmer 2006).

- The convection occurs in deep gaps, up to about $2 \mathrm{Mm}$ (Rempel et al. 2008) with strongly reduced field strength.

- The simulations show horizontal outflows, similar to Evershed flows but with smaller velocities, peaking near optical depth unity and associated with locally strongly inclined fields.

- The bright heads of the penumbra filaments show inward propagation and strong upflows

- The simulations show moving magnetic features (MMF's) and moat flow in the surrounding photosphere

For overturning convection to be efficient, the gas needs to stay near the surface for a significant amount of time in order to give it time to cool radiatively. At the same time, it needs to move away from its upflow point in order to allow more gas to flow up. Horizontal flows are thus essential components of overturning convection. The 


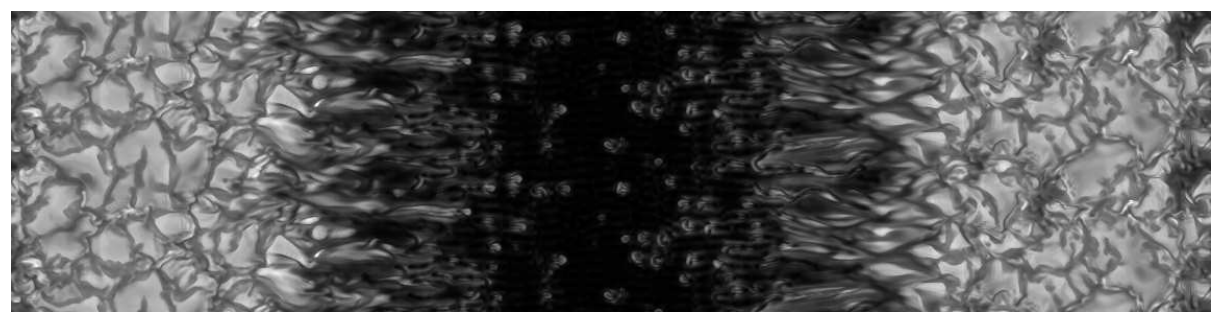

Fig. 7 Synthetic continuum image calculated from 3D MHD simulations, showing a sunspot and its surrounding photosphere (Rempel et al. 2008). In the umbral part of the spot is seen umbral dots with dark cores, in the penumbral part filaments that reach lengths of up to 2-3 Mm.

Evershed flow is identified as being identical to the horizontal flow component of this penumbral convection (Scharmer et al. 2008b).

The simulations reproduce fundamental properties of observed penumbrae. This gives us confidence in concluding that the simulations constitute good representations of penumbral dynamics and energy balance. However, neither of these penumbra simulations show nearly horizontal magnetic fields in the outer penumbra, as observed, so something is missing in the simulations. Other features that do not appear realistic are elongated structures in the outer penumbra that appear intermediate to granules and filaments and a less distinct boundary to the umbra than observed. A complex question concerns the field strengths in the gaps. In the simulations these are on the order of 700-1000 G. In the simulations of Heinemann et al. (2007), this was interpreted as a consequence of numerical diffusivities, allowing overturning convective flows to cross field lines. Similar processes take place in the simulations of Rempel et al. (Rempel, private communication). The existence of strong magnetic fields associated with overturning convection in penumbrae thus rely on turbulent magnetic diffusion at small scales. In contrast, umbral dots in the simulations of Rempel et al. (2008) as well as those of Schüssler \& Vögler (2006) appear as nearly field-free plumes near the surface.

The simulations of Rempel et al. show some indication of flow patterns that are reminiscent of roll convection (Danielson 1961), but this interpretation is uncertain. The convective energy transport is mainly provided by deep upflow and downflow plumes. An intriguing result of both simulations is that the convective gaps do not extend to the field-free atmosphere below the sunspot, suggesting that sunspots radiate energy that is contained within the magnetic field initially. A difference between the simulations of Rempel et al. and Heinemann et al. is that the former simulations show radial inflows adjacent to the outflows near the surface whereas the latter simulations show evidence of such return flows only deeper below the surface.

A perhaps relevant result for understanding penumbral magnetic fields comes from simulations of quiet sun emerging flux. In these simulations, the expulsion of magnetic flux takes place in the horizontal direction by horizontal flows but also in the vertical direction by overshooting convection (Steiner et al. 2008). The weak horizontal fields of such emerging flux are expelled to heights of about $500 \mathrm{~km}$, where they are difficult to observe in photospheric lines. Such expelled magnetic fields are limited to a height at which the gas pressure below the expelled magnetic field is roughly equal to $B^{2} / 2 \mu_{0}$. If a similar expulsion mechanism operates in the penumbra, where the field strength is much higher, any expelled horizontal field must be located much closer to the penum- 
bral photosphere than for the emerging flux discussed by Steiner et al. (2008). Such magnetized gas would then show strong polarization signatures reminiscent of flux tubes.

4.4 Connections to flux tube models

In spite of its $1 \mathrm{D}$ representation and the failure to confirm interchange convection, the moving tube model (Schlichenmaier et al. 1998a, b; Schlichenmaier 2002) has connections to convective processes. The upflow within the flux tube is driven by the superadiabatic stratification of the external atmosphere, similar to that of a field-free convective upflow. The flux tube evolution is similarly driven by the superadiabatic stratification of the external atmosphere, even though the complete cycle of inward/outward movement and heating/cooling of the flux tubes does not take place in the simulations: a flux tube initially located along the magnetopause moves toward the umbra and remains there. These 1D simulations cannot show convective flow patterns. However, the 'sea serpent' behavior (Schlichenmaier 2002), with upflows and downflows along the length of the tube, can be interpreted as a $1 \mathrm{D}$ representation of $3 \mathrm{D}$ convection. In the $3 \mathrm{D}$ penumbra simulations (Heinemann et al. 2007; Rempel et al. 2008), the upflows occur at the centers of the gaps and the downflows on either side of the upflows, displaced both the in the azimuthal and radially outward directions. In the moving tube model, such upflows and downflows can only be spatially separated in the radial direction. Both models have in common a convective upflow and radiative cooling driving an outflow away from the center of the spot. In both moving tube and 3D penumbra simulations, the outflow peaks in a thin layer near $\tau=1$, which is where the gas cools most efficiently (Scharmer et al. 2008b).

In the convective gap model, the strong magnetic field gradients above the gap are explained as a perturbation of a nearly potential magnetic field above the penumbra, introduced by the nearly field-free gap (Spruit \& Scharmer 2006; Scharmer \& Spruit 2006). This leads to a magnetic field that is cusp-shaped in the inner penumbra and locally nearly horizontal in the outer penumbra. Adding a weak horizontal magnetic inside the gap will not change this configuration significantly. Such a configuration is in its upper parts quite similar to a flux tube but deeper down, these two types of structures are very different.

Support for the existence of embedded flux tubes based on a magnetostatic model is claimed from calculations of net circular polarization (NCP), (Borrero et al. 2007). However, the $\tau=1$ surface of these models intersect the symmetry axis of the flux tube well above the center of the flux tube and the $\tau=0.1$ surface (typical of the line formation height) cuts through the top of the flux tube (c.f., Fig. 1). These calculations clearly are sensitive only to the upper part of the flux tube, where its magnetic field is similar to that of the convective gap model.

A similar ambiguity concerns the origin of the dark cores of penumbral filaments, explained by convective gap models (Spruit \& Scharmer 2006; Scharmer \& Spruit 2006). Also flux tube models with weaker field in the flux tube than in the surroundings produce opacity effects resulting in dark-cored structures Ruiz Cobo \& Bellot Rubio 2008). This is interpreted as support for flux tube models by the authors. However, also for this model, only the top of the flux tube is visible above $\tau=1$, so this configuration is similar to a convective gap model in its observable parts. 
Interpretations of highly resolved Stokes spectra SOT/Hinode show wrapping around structures that can similarly be interpreted either as flux tubes or as convecting gaps (Borrero et al. 2008). These and other recent high-resolution Stokes data do not provide evidence for flow channels and flux tubes elevated above the photosphere, as discussed in some papers, e.g., Solanki \& Montavon (1993); Rimmele (1995); Borrero et al. (2006). Based on observations of penumbra magnetic fields, interpretations in terms of convective gaps or flux tubes partly buried below the $\tau=1$ surface thus are inherently ambiguous. This also serves as a reminder of the difficulties of interpreting (inadequately resolved) observations in terms of unknown underlying physics.

While this ambiguity, in our opinion, undermines arguments for the very existence of embedded flux tubes, it primarily suggests that magnetic field measurements are not likely to show a clear distinction between flux tube and convective gap models, at least in the outer parts of the penumbra. The distinction between the models may need to be based primarily on measurements of the velocity field, which is horizontal and along a flux tube but with added vertical and azimuthal flow components in the convective gap models. It is this diagnostics that so far provides the strongest observational evidence in favor of the convecting gap model (Ichimoto et al. 2007). The observational evidence accumulated so far is however too scarce to be conclusive. Zakharov et al. (2008) also reported evidence for convective upflows in penumbral filaments, but clearly observations of the entire sequence of upflows, horizontal flows and downflows are needed to fully reveal the nature of convection in penumbrae.

A highly controversial issue is whether the penumbral convection is essentially fieldfree or associated with kG strengths. Bellot Rubio et al. (2007), based on SOT/Hinode data and Milne-Eddington (ME) inversions, found only small variations in field strength across dark-cored penumbral filaments. Scharmer et al. (2008a), based on SST observations and ME inversions, demonstrated that an improvement of the spatial resolution from 0.3 " to 0.15 " increases measured field strength variations over dark cores by approximately a factor of two. Zakharov et al. (2008), based on SST data and ME inversions, found locally weaker fields by approximately a factor two, associated with convecting filaments. Jurcák et al. (2007) using SOT/Hinode data and inversions allowing for gradients and a Gaussian perturbation in the magnetic field to represent flux tubes, concluded that the field strength is reduced by only $600 \mathrm{G}$ at the centers of bright filaments (where dark cores should be located) in the inner penumbra and furthermore that this reduction in field strength occurs only close to the photosphere and disappears already at $\log \tau=-0.5$. Based on observations and inversions, there is so far no support for the assumption that these gaps are nearly field-free. This conclusion refers to observational data from the layers above the photosphere whereas the nature of penumbral convection in deeper layers can ultimately only be determined from $3 \mathrm{D}$ MHD simulations.

\section{Conclusions}

We believe that there is now strong evidence to support the conclusion that penumbral fine structure should be interpreted as the result of overturning convection, as proposed by Spruit \& Scharmer (2006). Evidence for this conclusion comes from recent SOT/Hinode and SST observations (Ichimoto et al. 2007; Zakharov et al. 2008), showing vertical flows of the right magnitude to explain the penumbral radiative heat flux. Recent numerical 3D MHD simulations (Heinemann et al. 2007; Rempel et al. 2008) 
reproduce fundamental properties of observed penumbrae and confirm the convective origin of penumbral filaments. The simulations show that the nature of this convection takes place in gaps with up to $2 \mathrm{Mm}$ depth and that any roll-like convection (Danielson 1961; Thomas \& Weiss 2004; Rempel et al. 2008), if present, is of small importance (Rempel et al. 2008). The Evershed flow is interpreted to be identical to the horizontal flow component of this convection (Scharmer et al. 2008b). Such horizontal flows are necessary in order to cool hot upflows by radiation.

Neither observations nor simulations lead to the conclusion that this convection is nearly field-free, as suggested (Spruit \& Scharmer 2006). However, inferred field strengths from spectropolarimetric data are obviously limited to layers above the photosphere, whereas simulations rely on numerical diffusivities to prevent instabilities at scales corresponding to the grid separation. Other uncertainties relate to the outer parts of penumbrae where observations show nearly horizontal field and even field lines dipping down into the photosphere (Borrero \& Solanki 2008). Simulations do not show structures of this type. It appears likely that downward pumping of magnetic field by convection outside the sunspot plays a role in the outer penumbra, as proposed earlier (Thomas et al. 2002b), but we disagree strongly with the conclusion that this explains the origin of the filamentary structure of the penumbra. Downward pumping by the convection inside the penumbra also must take place and this probably explains why observations (Westendorp Plaza et al. 1997) show evidence of return flux well inside the outer penumbral boundary (Scharmer et al. 2008b).

Penumbral filaments have been successfully interpreted in terms of embedded flux tubes during a period of 15 years. While we conclude that this interpretation is misleading in terms of underlying physics, there are several reasons why this model has been so successful. We have shown that the opening of radially aligned gaps with nearly field-free convecting gas leads to a magnetic field that is much more horizontal over the gaps, giving the illusion of a flux tube (Spruit \& Scharmer 2006). There are also other arguments for expecting nearly horizontal fields in the penumbral atmosphere: Horizontal cooling flows are most efficient near optical depth unity and if this gas is magnetized, it will aid in producing nearly horizontal magnetic fields. Also, emerging flux simulations relevant to the quiet sun suggests that convection can lead to flux expulsion in the vertical direction in addition to the horizontal direction and that this explains quiet sun horizontal magnetic fields above the photosphere. Clear evidence of such 'vertical' flux expulsion is however not seen in the penumbral part of the sunspot simulated by Rempel et al. (2008).

We emphasize the connections of the moving tube model to convective processes and to the radiative cooling of such flows near the photosphere in both types of models. The similarity of the magnetic field above convecting gaps and flux tubes add to the difficulties of correctly interpreting observations and distinguishing between models.

In the embedded flux tube models, the Evershed flow is at center stage and the mechanism for heating the penumbra remains obscure. The new view of penumbral fine structure as caused by overturning convection implies that the main driver of penumbra fine structure is the energy flux below the surface and that the Evershed flow is 'only' a consequence of this convection (Scharmer et al. 2008b).

We believe that siphon flow models (Montesinos \& Thomas 1997) are of little relevance for understanding penumbrae. These are linked to the idea that there are two distinct families of field lines: those associated with dark filaments and Evershed flows and those associated with bright filaments connecting to distant magnetic regions (Weiss et al. 2004; Thomas \& Weiss 2004). As far as we know, there is no observa- 
tional support for this 'static' picture of penumbral magnetic fields. Observations suggest life times for penumbral filaments on the order of one hour associated with flow channels opening and closing continuously (Rimmele \& Marino 2006). 3D MHD Simulations (Rempel et al. 2008; Heinemann et al. 2007) confirm the transient nature of azimuthal variations in field strength and inclination. Theoretical arguments and models (Spruit \& Scharmer 2006; Scharmer \& Spruit 2006) as well as simulations clearly lead to the conclusion that the large variations in inclination across filaments are local perturbations, caused by penumbral convection and vanishing a few hundred $\mathrm{km}$ above the penumbral photosphere.

As regards further progress in this rapidly evolving field, we expect that even more realistic 3D MHD simulations in the near future will further improve our understanding of penumbrae, in particular as regards their outermost parts. Observed unpolarized and polarized spectra at the highest possible spatial resolution are needed. Of particular importance is such spectra giving information about the layer immediately above the photosphere. Emphasis should be given to analyzing data at +/- 90 deg from the symmetry axis of sunspots located away from disk center, as was done by Ichimoto et al. (2007). This is in part to allow analysis of flows perpendicular to the radial direction of the filaments, but also in order to see as deep into these structures as possible. Analysis of such data need to account for the pronounced 3D nature of these filaments, caused by strong azimuthal variations in the Wilson depression, as well as strong LOS variations in the magnetic field and flow velocity. A dilemma here is that the use of inversion techniques with many nodes along the LOS raises questions of uniqueness and difficulties in comparing the results of such inversions with simulations. Existing 3D MHD simulation data allow inversion techniques to be tested with synthetic Stokes spectra from penumbral atmospheres, as done already with simulations of small-scale flux concentrations outside sunspots (Khomenko \& Collados 2007; Orozco Suárez et al. 2007). The effect of assuming e.g. hydrostatic equilibrium can be evaluated quantitatively.

Presently used inversion techniques process polarized spectra pixel by pixel without constraining, for example, the magnetic field to be divergence-free. The requirement of divergence-free magnetic fields is crucial in forcing field lines to bend around convecting gaps (and flux tubes), leading to strong gradients in field strength and inclination. With spectropolarimetric observations approaching a spatial resolution of $100 \mathrm{~km}$ (Scharmer et al. 2008a), which is similar to the equivalent LOS resolution achieved with inversion techniques using a small number of nodes, it is reasonable to enforce magnetic fields constrained by $\operatorname{div}(\mathbf{B})=0$. Stray-light corrections are with most inversion techniques implemented in an ad-hoc manner pixel by pixel whereas a physical stray-light implementation would employ a point spread function that does not vary, or varies slowly, across the FOV. Micro- and macro-turbulence parameters are used as fudge parameters to compensate spatial smearing of unresolved structures. With improved spatial resolution, modeled LOS velocity gradients should eliminate the need for such parameters in the inversions. We expect future inversion techniques to develop as 'global' techniques, in the sense of fitting model parameters for a large number of connected pixels simultaneously. This will allow constraints, such as $\operatorname{div}(\mathbf{B})=0$, and physical straylight models to be incorporated in a consistent manner to further enhance the usefulness of inversion techniques for inferring the physical state of the atmospheres above sunspots and other magnetic structures. 
Acknowledgements The author gratefully acknowledges discussions with Henk Spruit, Åke Nordlund, Rolf Schlichenmaier, Matthias Rempel, Manfred Schüssler and Oskar Steiner. The author also thanks Rolf Schlichenmaier for valuable comments on the manuscript.

\section{References}

Bellot Rubio, L. R., Balthasar, H., \& Collados, M. 2004, A\&A, 427, 319

Bellot Rubio, L. R., Balthasar, H., Collados, M., \& Schlichenmaier, R. 2003, A\&A, 403, L47

Bellot Rubio, L. R., Schlichenmaier, R., \& Tritschler, A. 2006, A\&A, 453, 1117

Bellot Rubio, L. R., Tsuneta, S., Ichimoto, K., et al. 2007, ApJL, 668, L91

Bharti, L., Jain, R., \& Jaaffrey, S. N. A. 2007a, ApJL, 665, L79

Bharti, L., Joshi, C., \& Jaaffrey, S. N. A. 2007b, ApJL, 669, L57

Borrero, J. M. 2007, A\&A, 471, 967

Borrero, J. M., Bellot Rubio, L. R., \& Müller, D. A. N. 2007, ApJL, 666, L133

Borrero, J. M., Lagg, A., Solanki, S. K., \& Collados, M. 2005, A\&A, 436, 333

Borrero, J. M., Lites, B. W., \& Solanki, S. K. 2008, A\&A, 481, L13

Borrero, J. M. \& Solanki, S. K. 2008, ArXiv e-prints, 806

Borrero, J. M., Solanki, S. K., Bellot Rubio, L. R., Lagg, A., \& Mathew, S. K. 2004, A\&A, 422, 1093

Borrero, J. M., Solanki, S. K., Lagg, A., Socas-Navarro, H., \& Lites, B. 2006, A\&A, 450,383

Carlsson, M., Stein, R. F., Nordlund, Å., \& Scharmer, G. B. 2004, ApJL, 610, L137

Danielson, R. E. 1961, ApJ, 134, 289

Degenhardt, D. 1989, A\&A, 222, 297

-. 1991, A\&A, 248, 637

Heinemann, T. 2006, M.S. thesis, Univ. Copenhagen

Heinemann, T., Nordlund, Å., Scharmer, G. B., \& Spruit, H. C. 2007, ApJ, 669, 1390

Ichimoto, K., Suematsu, Y., Tsuneta, S., et al. 2007, Science, 318, 1597

Jahn, K. \& Schmidt, H. U. 1994, A\&A, 290, 295

Jurcák, J., Bellot Rubio, L., Ichimoto, K., et al. 2007, PASJ, 59, 601

Jurčák, J., Martínez Pillet, V., \& Sobotka, M. 2006, A\&A, 453, 1079

Keller, C. U., Schüssler, M., Vögler, A., \& Zakharov, V. 2004, ApJL, 607, L59

Khomenko, E. \& Collados, M. 2007, Memorie della Societa Astronomica Italiana, 78, 166

Langhans, K. 2006, in Astronomical Society of the Pacific Conference Series, Vol. 358, Astronomical Society of the Pacific Conference Series, ed. R. Casini \& B. W. Lites, $3-+$

Langhans, K., Scharmer, G. B., Kiselman, D., \& Löfdahl, M. G. 2007, A\&A, 464, 763

Leka, K. D. 1997, ApJ, 484, 900

Lites, B. W., Elmore, D. F., Seagraves, P., \& Skumanich, A. P. 1993, ApJ, 418, 928

Lites, B. W., Scharmer, G. B., Berger, T. E., \& Title, A. M. 2004, Sol. Phys., 221, 65

Martínez Pillet, V. 2000, A\&A, 361, 734

Martínez Pillet, V., Collados, M., Bellot Rubio, L. R., et al. 1999, in Astronomische Gesellschaft Abstract Series, Vol. 15, Astronomische Gesellschaft Abstract Series, ed. R. E. Schielicke, 89-+

Meyer, F. \& Schmidt, H. U. 1968, Mitteilungen der Astronomischen Gesellschaft Hamburg, 25, 194 
Montesinos, B. \& Thomas, J. H. 1989, ApJ, 337, 977

-. 1993, ApJ, 402, 314

-. 1997, Nature, 390, 485

Nordlund, A. 2006, in Astronomical Society of the Pacific Conference Series, Vol. 354, Solar MHD Theory and Observations: A High Spatial Resolution Perspective, ed. J. Leibacher, R. F. Stein, \& H. Uitenbroek, 353-+

Orozco Suárez, D., Bellot Rubio, L. R., \& del Toro Iniesta, J. C. 2007, ApJL, 662, L31 Rempel, M., Schuessler, M., \& Knoelker, M. 2008, ArXiv e-prints

Rezaei, R., Schlichenmaier, R., Beck, C., \& Bellot Rubio, L. R. 2006, A\&A, 454, 975

Riethmüller, T. L., Solanki, S. K., \& Lagg, A. 2008, ApJL, 678, L157

Rimmele, T. 2008, ApJ, 672, 684

Rimmele, T. \& Marino, J. 2006, ApJ, 646, 593

Rimmele, T. R. 1995, A\&A, 298, 260

Rouppe van der Voort, L. H. M. 2002, A\&A, 389, 1020

Ruiz Cobo, B. \& Bellot Rubio, L. R. 2008, A\&A, 488, 749

Sainz Dalda, A. \& Bellot Rubio, L. R. 2008, A\&A, 481, L21

Sams, III, B. J., Golub, L., \& Weiss, N. O. 1992, ApJ, 399, 313

Sanchez Almeida, J. \& Lites, B. W. 1992, ApJ, 398, 359

Scharmer, G. B., Gudiksen, B. V., Kiselman, D., Löfdahl, M. G., \& Rouppe van der Voort, L. H. M. 2002, Nature, 420, 151

Scharmer, G. B., Langhans, K., Kiselman, D., \& Löfdahl, M. G. 2007, in Astronomical Society of the Pacific Conference Series, Vol. 369, New Solar Physics with Solar-B Mission, ed. K. Shibata, S. Nagata, \& T. Sakurai, 71-+

Scharmer, G. B., Narayan, G., Hillberg, T., et al. 2008a, ArXiv e-prints

Scharmer, G. B., Nordlund, Å., \& Heinemann, T. 2008b, ApJL, 677, L149

Scharmer, G. B. \& Spruit, H. C. 2006, A\&A, 460, 605

Schlichenmaier, R. 2002, Astronomische Nachrichten, 323, 303

Schlichenmaier, R. 2003, in Astronomical Society of the Pacific Conference Series, Vol. 286, Current Theoretical Models and Future High Resolution Solar Observations: Preparing for ATST, ed. A. A. Pevtsov \& H. Uitenbroek, 211-+

Schlichenmaier, R., Bruls, J. H. M. J., \& Schüssler, M. 1999, A\&A, 349, 961

Schlichenmaier, R. \& Collados, M. 2002, A\&A, 381, 668

Schlichenmaier, R., Jahn, K., \& Schmidt, H. U. 1998a, ApJL, 493, L121+

-. 1998b, A\&A, 337, 897

Schlichenmaier, R. \& Solanki, S. K. 2003, A\&A, 411, 257

Schüssler, M. \& Vögler, A. 2006, ApJL, 641, L73

Solanki, S. K. \& Montavon, C. A. P. 1993, A\&A, 275, 283

Solanki, S. K., Montavon, C. A. P., \& Livingston, W. 1994, A\&A, 283, 221

Solanki, S. K., Walther, U., \& Livingston, W. 1993, A\&A, 277, 639

Spruit, H. C. 1976, Sol. Phys., 50, 269

Spruit, H. C. \& Scharmer, G. B. 2006, A\&A, 447, 343

Steiner, O. 2005, A\&A, 430, 691

Steiner, O., Rezaei, R., Schaffenberger, W., \& Wedemeyer-Böhm, S. 2008, ApJL, 680, L85

Thomas, J. H. 1988, ApJ, 333, 407

-. 2005, A\&A, 440, L29

Thomas, J. H. 2006, in Astronomical Society of the Pacific Conference Series, Vol. 354, Solar MHD Theory and Observations: A High Spatial Resolution Perspective, ed. J. Leibacher, R. F. Stein, \& H. Uitenbroek, 224-+ 
Thomas, J. H. \& Montesinos, B. 1990, ApJ, 359, 550

-. 1991, ApJ, 375, 404

Thomas, J. H. \& Weiss, N. O. 2004, ARA\&A, 42, 517

Thomas, J. H., Weiss, N. O., Tobias, S. M., \& Brummell, N. H. 2002a, Nature, 420, 390

—. 2002b, Astronomische Nachrichten, 323, 383

Tritschler, A., Müller, D. A. N., Schlichenmaier, R., \& Hagenaar, H. J. 2007, ApJL, 671, L85

van Noort, M. J. \& Rouppe van der Voort, L. H. M. 2008, A\&A, 489, 429

Weiss, N. O. 2002, Astronomische Nachrichten, 323, 371

-. 2006a, Space Science Reviews, 124, 13

Weiss, N. O. 2006b, in Astronomical Society of the Pacific Conference Series, Vol. 354, Solar MHD Theory and Observations: A High Spatial Resolution Perspective, ed. J. Leibacher, R. F. Stein, \& H. Uitenbroek, 213-+

Weiss, N. O., Thomas, J. H., Brummell, N. H., \& Tobias, S. M. 2004, ApJ, 600, 1073

Westendorp Plaza, C., del Toro Iniesta, J. C., Ruiz Cobo, B., et al. 1997, Nature, 389, 47

Zakharov, V., Hirzberger, J., Riethmüller, T. L., Solanki, S. K., \& Kobel, P. 2008, A\&A, 488, L17 